Review Article

\title{
New Developments in Peritoneal Fibroblast Biology: Implications for Inflammation and Fibrosis in Peritoneal Dialysis
}

\author{
Janusz Witowski, ${ }^{1,2}$ Edyta Kawka, ${ }^{1,2}$ Andras Rudolf, ${ }^{1}$ and Achim Jörres ${ }^{2}$ \\ ${ }^{1}$ Department of Pathophysiology, Poznan University of Medical Sciences, Medical Biology Centre, Rokietnicka 8, \\ 60-806 Poznan, Poland \\ ${ }^{2}$ Department of Nephrology and Medical Intensive Care, Charité-Universitätsmedizin Berlin, Campus Virchow-Klinikum, \\ Augustenburger Platz 1, 13353 Berlin, Germany
}

Correspondence should be addressed to Janusz Witowski; jwitow@ump.edu.pl

Received 22 June 2015; Revised 11 August 2015; Accepted 25 August 2015

Academic Editor: Gang Liu

Copyright (c) 2015 Janusz Witowski et al. This is an open access article distributed under the Creative Commons Attribution License, which permits unrestricted use, distribution, and reproduction in any medium, provided the original work is properly cited.

Uraemia and long-term peritoneal dialysis (PD) can lead to fibrotic thickening of the peritoneal membrane, which may limit its dialytic function. Peritoneal fibrosis is associated with the appearance of myofibroblasts and expansion of extracellular matrix. The extent of contribution of resident peritoneal fibroblasts to these changes is a matter of debate. Recent studies point to a significant heterogeneity and complexity of the peritoneal fibroblast population. Here, we review recent developments in peritoneal fibroblast biology and summarize the current knowledge on the involvement of peritoneal fibroblasts in peritoneal inflammation and fibrosis.

\section{Introduction}

Fibroblasts are the commonest connective tissue cells and the main source of extracellular matrix. Until recently, fibroblasts have been viewed as cells providing only structural framework for tissues. Now it is clear that fibroblasts are at the center of tissue homeostasis and serve specialized functions in different organs. Impressive versatility of fibroblasts is reflected by differences in gene expression patterns according to anatomic location [1]. Moreover, even the same tissue can be populated with several fibroblast subsets with distinct functions [2]. The phenotype of fibroblasts may change further during wound healing or fibrosis, when cells become activated and termed "myofibroblasts." When analyzing fibroblasts, it is therefore essential to take the exact physiological and clinical context into account. Here, we review new developments in our understanding of the role of fibroblasts in the peritoneum, especially their involvement in peritoneal dialysis- (PD-) associated fibrosis.

\section{Fibroblast Identity and Phenotype}

Normal resident tissue fibroblasts are identified by their spindle-shape appearance and location within the connective tissue. They may also express fibroblast-specific protein-1 (FSP-1), but not molecular markers for other cell types. In response to tissue injury and stimulation with growth factors (e.g., TGF- $\beta$ ), fibroblasts can adopt an activated phenotype resembling that of smooth muscle cells and characterized by the expression of smooth muscle actin $(\alpha$-SMA). The resulting myofibroblasts have increased contractile capacity and show increased proliferation and motility. They produce more extracellular matrix (ECM) components and more regulators of the ECM turnover. The origin of myofibroblasts in different tissues (including the peritoneum) is a matter of intensive research and some controversy. Classically, myofibroblasts were thought to derive from resident tissue fibroblasts. However, there are other potential precursors of myofibroblasts, including epithelial, mesothelial, and endothelial cells, as well as bone marrow-derived fibrocytes. 
Introduction of new fibroblast biomarkers and great advances in lineage tracing techniques allowed us to better define the contribution of these cells to adverse tissue remodeling.

The analysis of peritoneal membrane biopsies from patients treated with PD has clearly demonstrated that the thickness of the submesothelial compact zone, a layer of mature fibrous tissue containing collagen and elastin fibers, progressively increases with duration of $\mathrm{PD}$ [3]. This is particularly evident in the parietal peritoneum [4]. Thickening of the peritoneum reflects the expansion of ECM, which is deposited primarily by myofibroblasts. To determine the origin of myofibroblasts, it is necessary to identify first resident fibroblasts and mesothelial cells. This has not always been an easy task, either in vivo or in culture (see [5] for a review). Mesothelial cells display typically an epithelial-like appearance and form a monolayer covering the peritoneal surface, while fibroblasts are fusiform cells embedded in the submesothelial interstitium. In the course of mesenchymal transition, however, mesothelial cells acquire a fibroblastlike phenotype, become motile, and invade the underlying stroma. Moreover, both fibroblasts and mesothelial arise from the mesoderm and can share rather than differ in certain biomarkers, for example, vimentin. Therefore, the identification of FSP-1, a calcium-binding protein of the S100 family, as providing better specificity for fibroblasts $[6,7]$ was viewed as a great step forward in tracking fibroblasts. However, the validity of FSP-1 as the specific fibroblast marker has been questioned. It turned out that FSP-1 might be absent in a proportion of normal interstitial fibroblasts and some FSP-1-positive cells in diseased tissues might be, in fact, mononuclear or endothelial cells [8]. Despite these uncertainties, FSP-1 in combination with other biomarkers is still used in studies on the origin of peritoneal myofibroblasts $[9,10]$. In cell culture, antibodies against FSP-1 can help purify peritoneal fibroblasts from contaminating mesothelial cells [11] (Figure 1).

\section{Fibroblast Subsets}

In addition to FSP-1, further fibroblast subsets can be identified using other criteria. Thy-1 (CD90), a glycophosphatidylinositol-linked outer cell membrane protein, is expressed by many cell types, but it can separate fibroblasts into Thy$1^{+}$and Thy- $1^{-}$subpopulations with different phenotypic and functional features [12-15]. The significance of this trait may differ according to the origin of fibroblasts. Normal lung fibroblasts, both in mice and in humans, are predominantly Thy-1-positive and their presence appears to limit pulmonary fibrosis [15-18]. It has been demonstrated that the absence of Thy-1 on lung fibroblasts is associated with their myofibroblastic phenotype and enhanced proliferative response to fibrogenic stimuli. Mice deficient in Thy-1 show exaggerated fibrosis and myofibroblastic differentiation after bleomycininduced pulmonary injury. In humans with idiopathic pulmonary fibrosis, no Thy-1 staining was seen in fibroblastic foci. These results suggest that the loss of lung fibroblast Thy1 expression after injury promotes enhanced fibrogenesis. In contrast to lung fibroblasts, however, the myofibroblastic conversion of orbital and myometrial fibroblasts in response to TGF- $\beta$ appears to be favored by the presence rather than the absence of Thy-1 [12].

Little is known about the role of Thy-1 in peritoneal cells. A small population of Thy $1^{+}\left(\mathrm{CD}^{+} 0^{+}\right)$mesotheliallike cells has recently been detected in ascites drained from patients with gastrointestinal cancers [19]. These cells were defined as mesenchymal stem cells and showed a distinct myofibroblastic phenotype after stimulation with TGF- $\beta$. We have examined Thy-1 expression patterns in apparently normal human peritoneal fibroblasts (HPFB) in culture. Indeed, it appears that both Thy $-1^{+}$and Thy- $1^{-}$subsets of HPFB exist in the peritoneum and differ in morphology and the ability to acquire a myofibroblastic phenotype (Kawka E. et al., personal observations).

\section{Resident Peritoneal Fibroblasts}

Fibroblasts of the normal peritoneum are scattered in the submesothelial connective tissue. Electron microscopy shows large multipolar cells embedded between collagen and elastic fibers [20]. These cells express neither myofibroblastic nor mesothelial markers [21]. Also, the expression of FSP-1 is not evident $[9,22]$ (Kawka E. et al., personal observations), which adds to the reservations about FSP-1 marking resident fibroblasts in the normal peritoneum [8]. On the other hand, the cells may bear platelet-derived growth factor receptor$\beta$ (PDGFR $\beta, \mathrm{CD} 140 \mathrm{~b})[23]$, which is sometimes used as a marker of resident fibroblasts. However, PDGFR $\beta$ is also expressed by pericytes and the exact relationship between pericytes and perivascular fibroblasts is not clear [24].

The cells identified as submesothelial fibroblasts occasionally express hematopoietic cell surface marker CD34 [21], which may indicate that they are derived from blood-borne fibrocytes. But they do not usually express other fibrocyte markers (CD45, CD11b, and MHC class II), suggesting that they are rather primal mesenchymal cells [25] residing in the peritoneum.

The thickness of the submesothelial compact zone in uremic patients is already increased before the commencement of dialysis, pointing to a detrimental impact of uraemia itself $[3,26]$. Nevertheless, the phenotype of resident fibroblasts and their biomarker expression patterns do not seem to be altered significantly [21]. PD exposure leads to further thickening of the compact zone and distinct changes in peritoneal fibroblasts. The presence of FSP-1 expression becomes evident $[9,10]$, although it is not clear whether this comes from resident fibroblasts or other cell types transitioning into fibroblasts (see below). Soon after the initiation of PD many fibroblasts acquire a myofibroblastic phenotype as evidenced by $\alpha$-SMA expression $[21,27,28]$. They often form clusters and localize immediately beneath the mesothelial surface. They may also lose CD34 expression and show cytokeratin and E-cadherin expression instead [21]. The significance of CD34 loss is unclear, although it has been observed in fibrotic lesions in other tissues [29]. On the other hand, the expression of cytokeratin and E-cadherin by myofibroblasts is viewed as an indication of their origin from mesothelial cells [30]. 


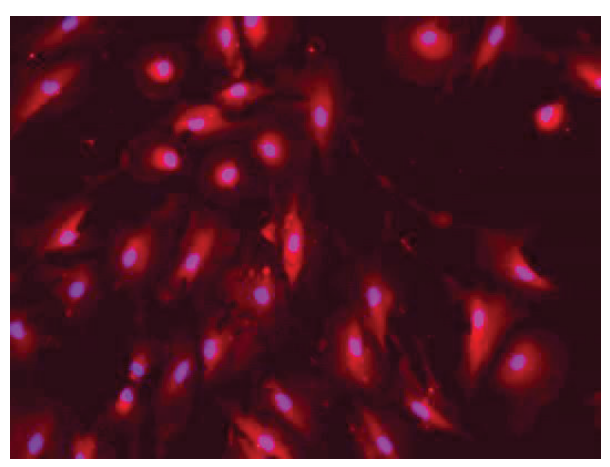

(a)

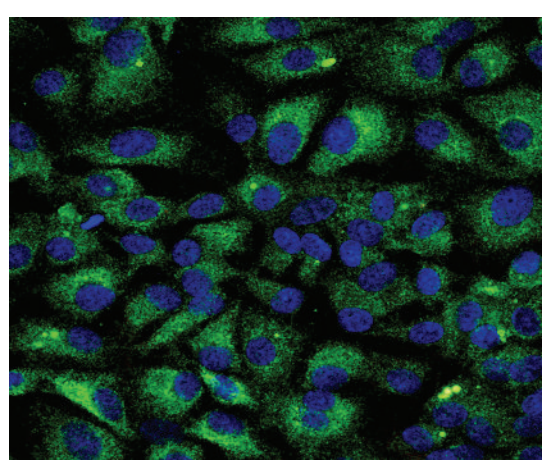

(b)

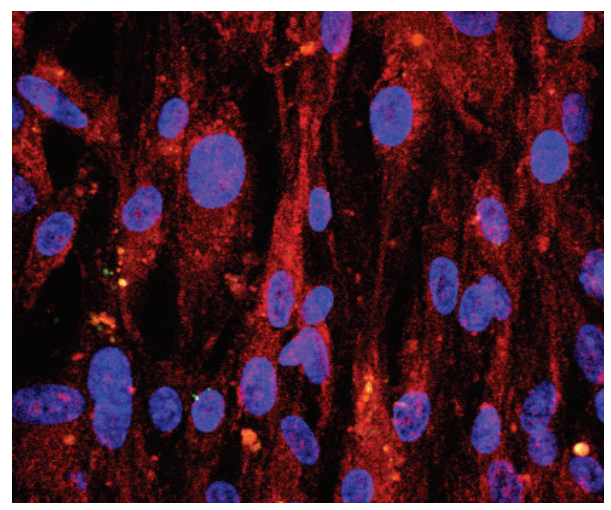

(c)

FIGURE 1: Expression of FSP-1 by human peritoneal fibroblasts and mesothelial cells. Human peritoneal mesothelial cells and peritoneal resident fibroblasts were isolated from apparently normal omentum by enzymatic digestion, as described [5]. Populations of mesothelial cells and fibroblasts were immunostained for FSP-1 (red) and cytokeratin (green). Nuclei were counterstained with DAPI (blue). Magnification 200x. (a) Human peritoneal fibroblasts express FSP-1 but not cytokeratin. (b) Human peritoneal mesothelial cells express cytokeratin but not FSP-1. (c) Stimulation of mesothelial cells with TGF- $\beta 1$ ( $1 \mathrm{ng} / \mathrm{mL} ; 72$ hours) leads to mesenchymal transition that is associated with loss of cytokeratin expression and de novo FSP-1 expression.

\section{Epithelial-to-Mesenchymal Transition as a Source of Peritoneal Fibroblasts}

The concept of peritoneal myofibroblasts arising from the mesothelium through epithelial-to-mesenchymal transition (EMT) received a lot of attention over the past decade (see [25, 31-33] for excellent reviews). It appears that in the course of $\mathrm{PD}$ mesothelial cells upregulate transcription factors of the snail, ZEB, and Twist families, which control molecular reprogramming of EMT [34]. The whole process can be induced by a number of growth factors, including transforming growth factor- $\beta$ (TGF- $\beta$ ), which has been identified as a key mediator of mesothelial EMT both in vitro [35] (Figure 1) and in vivo [36]. TGF- $\beta$ exerts its effects by engaging various members of the family of mitogen-activated protein kinases (MAPKs), including TGF- $\beta$-activated kinase1 (TAK-1) [37], p38 [38], and JNK kinase [39]. Inhibition of MAPK phosphorylation with glucocorticoids can block TGF- $\beta$-induced EMT of mesothelial cells in culture [40]. In addition to MAPKs, the involvement of the nuclear transcription factor $-\kappa \mathrm{B}(\mathrm{NF}-\kappa \mathrm{B})$ in mesothelial cell transition has been postulated [41]. In vivo, adenovirus-mediated overexpression of TGF- $\beta$ in the rat peritoneum results in the upregulation of several genes involved in EMT (snail, collagen 1, and $\alpha$-SMA) [36]. In this setting the cytokeratin/ $\alpha$-SMA double-positive cells appear first in the mesothelial monolayer and later in the reorganized submesothelial matrix [36]. These effects are mediated by both SMAD3-dependent and SMAD3independent mechanisms [42]. The fibroblast-like phenotype of mesothelial cells isolated from PD patients or treated in vitro with TGF- $\beta$ could be reversed by bone morphogenic protein-7 (BMP-7) [43] or by TAK-1 inhibitors [37]. Of particular interest in the context of $\mathrm{PD}$ is the observation that exposure of mesothelial cells in vitro to high glucose can induce Twist, a key EMT-controlling transcription factor [44], and increase the expression of $\alpha$-SMA [45]. These effects could be related to upregulation of TGF- $\beta$ by high glucose [46], but they can also be attributed to decreased expression of BMP-7 [45] or heme oxygenase-1 (HO-1) [47]. Indeed, experimental upregulation of BMP-7 or HO-1 could partly reduce high glucose-induced EMT of mesothelial cells. In an animal model of $\mathrm{PD}$, adenovirus-mediated transfection of BMP-7 was found to inhibit EMT in mesothelial cells and decrease subsequent peritoneal thickening [45].

What still remains unclear is the mechanism by which $P D$ exposure initiates EMT. The analysis of peritoneal membrane 
biopsies revealed that the loss of mesothelial cells from the peritoneal surface and the appearance of submesothelial cytokeratin staining occur relatively often and early during PD [49]. The observation that the number of fibroblastlike mesothelial cells isolated from spent dialysate effluent increases with the duration of therapy [30] points to the role of cumulative exposure to $\mathrm{PD}$ fluids and/or occasional episodes of peritonitis. In this respect, it has been demonstrated that key proinflammatory cytokines IL-1 $\beta$ and TNF $\alpha$ induce increased peritoneal TGF- $\beta$ expression in animal models [50]. Moreover, there exists data to suggest a link between EMT and biocompatibility of PD solutions. It has been observed that PD fluids with high concentration of glucose degradation products (GDP) can induce EMT in mesothelial cells both during short-term direct exposure in culture and-to lesser extent-after chronic PD exposure in vivo $[51,52]$. This finding is in line with earlier observations of EMT in the peritoneal membrane of rats treated with chronic intraperitoneal administration of GDP [53].

Loureiro and colleagues have assessed the exact contribution of various precursors to the pool of peritoneal fibroblasts that accumulate in the peritoneum of mice during chronic PD [9]. They found no FSP-1 ${ }^{+}$cells in the normal peritoneum. However, such cells did appear after exposure to PD fluids and could be further characterized by dual-immunolabeling using an anti-FSP-1 antibody in conjunction with antibodies against cytokeratin, CD45, or CD31. Approximately 37\% FSP$1^{+}$cells were identified as derived from mesothelial cells (FSP$1^{+} /$cytokeratin $^{+}$), $34 \%$ from fibrocytes $\left(\mathrm{FSP}-1^{+} / \mathrm{CD} 45^{+}\right)$, and $5 \%$ from endothelial cells $\left(\mathrm{FSP}-1^{+} / \mathrm{CD} 31^{+}\right)$. The remaining $24 \%$ cells stained singly for FSP-1 and their origin was not defined. Interestingly, by using three-color immunofluorescence it has been estimated that approximately 50\% of FSP$1^{+} /$cytokeratin $^{+}$cells coexpressed $\alpha$-SMA, which pointed to their myofibroblastic phenotype. Importantly, the administration of TGF- $\beta$-blocking peptides significantly reduced the extent of PD fluid-induced peritoneal fibrosis and the number of FSP- $1^{+}$cells, especially of the FSP- $1^{+} /$cytokeratin $^{+}$subpopulation. More recently, the same group have demonstrated that TGF- $\beta$ can be also involved in mesenchymal transition of mesothelial cells induced by endothelin-1 [54]. These data support the concept that peritoneal fibrosis in PD is largely related to TGF- $\beta$-driven conversion of mesothelial cells into myofibroblasts.

The potential of mesothelial cells to undergo EMT and to contribute to other cell lineages has been well documented during development. In particular, it has been demonstrated that the pleural mesothelium is a source of peribronchiolar fibroblasts in the foetal lung and that the process of mesothelial cell migration into the lung parenchyma is controlled by the hedgehog signaling pathway [55]. Moreover, it appears that such a process may also occur in adult tissues. In this respect, mesothelial cells covering the liver were found to differentiate into hepatic myofibroblasts during liver injury and fibrosis [56]. Similarly, EMT in pleural mesothelial cells was found to be contributing to idiopathic pulmonary fibrosis [57]. Interestingly, several of the above studies employed the Cre recombinase technology to trace the fate of mesothelial cells that expressed the Wilms tumor-1 (Wt-1) transcription factor as a biomarker. Genetic mapping of $\mathrm{Wt}-1^{+}$cells has also been used recently by Chen and colleagues to identify the cellular origin of myofibroblasts during peritoneal fibrosis [58]. The results of their study posed a challenge to the relevance of mesothelial cells as a source of peritoneal myofibroblasts. In contrast to earlier studies, they have observed that these were submesothelial resident fibroblasts rather than mesothelial cells that gave rise to collagen-producing myofibroblasts after injury induced by sodium hypochlorite (and to lesser extent by PD solutions or by adenovirus-mediated TGF$\beta 1$ overexpression). Resident fibroblasts were defined as cytokeratin $^{-}$, vimentin ${ }^{+}$, and $\operatorname{PDGF} \beta^{+}$cells located beneath mesothelial cell basement membrane. Interestingly, the use of PDGFR inhibitor after injury significantly attenuated the accumulation of $\alpha \mathrm{SMA}^{+}$myofibroblasts and peritoneal fibrosis. Obviously, the results of this study will need to be independently confirmed, given the limitations of current lineage tracing techniques. As correctly pointed out in a recent review [32], these technical shortcomings do not permit the possibility of mesothelial cell transition in vivo to be ruled out entirely. Moreover, mesothelial cells may still contribute to peritoneal fibrosis through their capacity for producing collagen [58]. They can also act indirectly by affecting peritoneal fibroblasts in a paracrine manner. In this respect, it has been demonstrated that lysophosphatidic acid signaling through $\mathrm{LPA}_{1}$, a G protein-coupled receptor, stimulates mesothelial cells to produce connective tissue growth factor (CTGF) that subsequently drives peritoneal fibrosis by inducing peritoneal fibroblast proliferation and collagen synthesis [59].

\section{Fibroblast Involvement in Peritoneal Inflammation}

Persistent tissue irritation, inflammation, and fibroblast activation are key features of fibrosis [60]. In this setting fibroblasts not only are effector cells but also contribute signals that control the function of other cell types. Peritonitis is the commonest PD-associated insult to the peritoneum, which is characterized by massive leukocyte infiltration. It is now recognized that the sequence at which different leukocyte subsets arrive in the peritoneum is controlled by a complex intraperitoneal network of chemokines [31]. These chemokines are thought to be produced primarily by mesothelial cells. However, peritoneal fibroblasts are also capable of synthesizing some chemokines and can be as potent in this respect as the mesothelium [61-63]. This activity of peritoneal fibroblasts can help recruit leukocytes during those episodes of severe peritonitis that are associated with extensive mesothelial cell damage and exfoliation [64, 65]. We have demonstrated that HPFB release chemokines MCP-1/CCL2 and IL-8/CXCL8 either constitutively or after stimulation with IL- $1 \beta$ and $\mathrm{TNF} \alpha$. The response to these macrophage-derived proinflammatory cytokines is mediated through transcription factors of the NF- $\kappa \mathrm{B}$ family [61]. The production of neutrophil-targeting cytokines by HPFB is triggered predominantly by IL-1 $\beta$ [62]. We have demonstrated that it stimulates the secretion of CXCL1 and CXCL8, classic chemokines for neutrophils, but also of granulocyte 


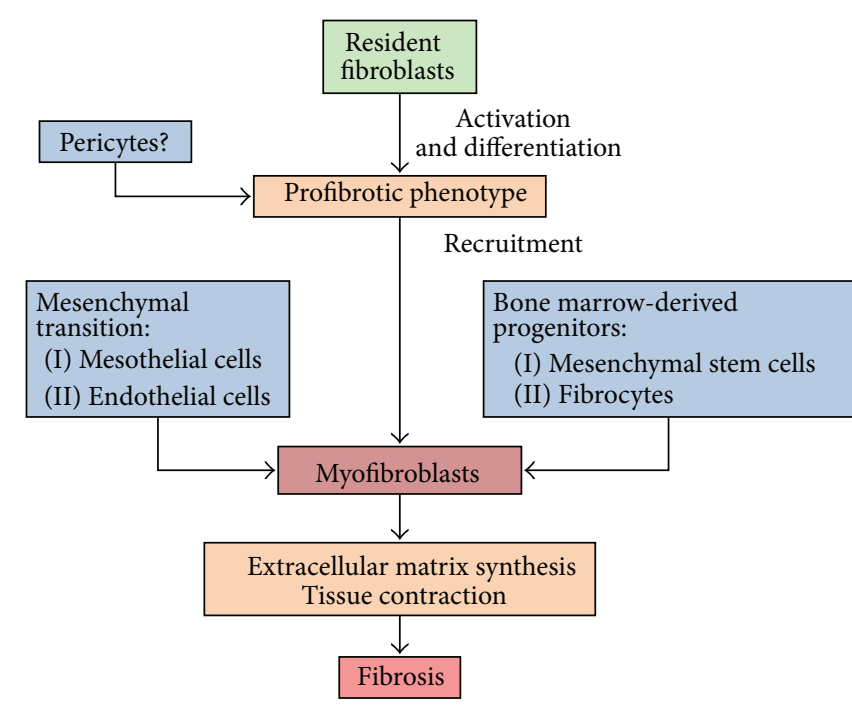

FIGURE 2: Potential myofibroblast precursors contributing to peritoneal fibrosis in PD. In response to peritoneal irritation or injury, myofibroblasts accumulate in the peritoneum as a result of (i) resident peritoneal fibroblasts activation and proliferation; (ii) transformation of local pericytes; (iii) proliferation and infiltration by resident and circulating fibrocytes; (iv) differentiation of local mesenchymal stem cells; (v) dedifferentiation and mesenchymal transition of peritoneal mesothelial cells; and (vi) dedifferentiation and mesenchymal transition of endothelial cells (adapted from [32, 48]).

colony-stimulating factor (G-CSF) that mobilizes neutrophils from the bone marrow and promotes their survival. On the other hand, we have demonstrated that HPFB can also produce CCL5, a strong chemoattractant for mononuclear leukocytes [63]. The process is critically controlled by IFN$\gamma$, which does not stimulate CCL5 itself but synergistically amplifies the effect of TNF $\alpha$. Moreover, by inducing CD40 expression it allows HPFB to synthesize CCL5 in response to CD40 ligand (CD40L) present primarily on T cells.

HPFB can also generate chemokines in response to high glucose exposure. It has recently been observed that the incubation of HPFB with glucose led to a dose-dependent increase in CCL2 mRNA expression [66]. It was preceded by a short-lived increase in the expression of the osmosensitive transcription factor, nuclear factor of activated $\mathrm{T}$ cells 5 (NFAT5). However, it is uncertain whether this glucoseinduced increase in CCL2 mRNA is mediated by NFAT5. The analysis of peritoneal biopsies revealed no difference in the expression of NFAT5 but still increased expression of CCL2 in the peritoneum of patients undergoing PD compared to those with uraemia but not requiring dialysis.

Taken together, all these observations are in line with the concept of resident tissue fibroblasts acting as sentinel cells that control inflammatory response to tissue injury or infection [67]. In this respect, HPFB can add significantly to transperitoneal chemotactic gradients during peritonitis.

\section{Conclusions}

Increasing data suggests that collagen-producing myofibroblasts that accumulate in the peritoneum in the course of
PD are derived from various precursors (Figure 2). The exact contribution of these cellular sources remains to be established. Of those, resident peritoneal fibroblasts still need to be considered as important predecessors of myofibroblasts. However, given unique immune environment of the peritoneal cavity, site-specific variation in fibroblast transcriptional profiles, and internal heterogeneity, it is essential to better understand the basics of peritoneal fibroblast biology. This is the prerequisite for interventional therapies targeting PD-associated peritoneal fibrosis.

\section{Conflict of Interests}

The authors declare that there is no conflict of interests regarding the publication of this paper.

\section{References}

[1] J. L. Rinn, C. Bondre, H. B. Gladstone, P. O. Brown, and H. Y. Chang, "Anatomic demarcation by positional variation in fibroblast gene expression programs," PLoS Genetics, vol. 2, no. 7, article el19, 2006.

[2] R. R. Driskell and F. M. Watt, "Understanding fibroblast heterogeneity in the skin," Trends in Cell Biology, vol. 25, no. 2, pp. 92-99, 2015.

[3] J. D. Williams, K. J. Craig, N. Topley et al., "Morphologic changes in the peritoneal membrane of patients with renal disease," Journal of the American Society of Nephrology, vol. 13, no. 2, pp. 470-479, 2002.

[4] J. D. Williams, K. J. Craig, C. Von Ruhland, N. Topley, and G. T. Williams, "The natural course of peritoneal membrane biology during peritoneal dialysis," Kidney International, Supplement, vol. 64, no. 88, pp. S43-S49, 2003.

[5] J. Witowski and A. Jörres, "Peritoneal cell culture: fibroblasts," Peritoneal Dialysis International, vol. 26, no. 3, pp. 292-299, 2006.

[6] F. Strutz, H. Okada, C. W. Lo et al., "Identification and characterization of a fibroblast marker: FSP1," Journal of Cell Biology, vol. 130, no. 2, pp. 393-405, 1995.

[7] R. Kalluri and E. G. Neilson, "Epithelial-mesenchymal transition and its implications for fibrosis," The Journal of Clinical Investigation, vol. 112, no. 12, pp. 1776-1784, 2003.

[8] M. Le Hir, I. Hegyi, D. Cueni-Loffing, J. Loffing, and B. Kaissling, "Characterization of renal interstitial fibroblastspecific protein 1/S100A4-positive cells in healthy and inflamed rodent kidneys," Histochemistry and Cell Biology, vol. 123, no. 4-5, pp. 335-346, 2005.

[9] J. Loureiro, A. Aguilera, R. Selgas et al., "Blocking TGF$\beta 1$ protects the peritoneal membrane from dialysate-induced damage," Journal of the American Society of Nephrology, vol. 22, no. 9, pp. 1682-1695, 2011.

[10] J. Loureiro, M. Schilte, A. Aguilera et al., "BMP-7 blocks mesenchymal conversion of mesothelial cells and prevents peritoneal damage induced by dialysis fluid exposure," Nephrology Dialysis Transplantation, vol. 25, no. 4, pp. 1098-1108, 2010.

[11] R. Catar, J. Witowski, P. Wagner et al., “The proto-oncogene CFos transcriptionally regulates VEGF production during peritoneal inflammation," Kidney International, vol. 84, no. 6, pp. 1119-1128, 2013. 
[12] L. Koumas, T. J. Smith, S. Feldon, N. Blumberg, and R. P. Phipps, "Thy-1 expression in human fibroblast subsets defines myofibroblastic or lipofibroblastic phenotypes," American Journal of Pathology, vol. 163, no. 4, pp. 1291-1300, 2003.

[13] L. Koumas and R. P. Phipps, "Differential COX localization and PG release in Thy- $1^{+}$and Thy-1- human female reproductive tract fibroblasts," The American Journal of Physiology: Cell Physiology, vol. 283, no. 2, pp. C599-C608, 2002.

[14] T. J. Smith, L. Koumas, A. Gagnon et al., "Orbital fibroblast heterogeneity may determine the clinical presentation of thyroidassociated ophthalmopathy," Journal of Clinical Endocrinology and Metabolism, vol. 87, no. 1, pp. 385-392, 2002.

[15] Y. Zhou, J. S. Hagood, and J. E. Murphy-Ullrich, “Thy-1 expression regulates the ability of rat lung fibroblasts to activate transforming growth factor-beta in response to fibrogenic stimuli," American Journal of Pathology, vol. 165, no. 2, pp. 659669, 2004.

[16] Y. Y. Sanders, P. Kumbla, and J. S. Hagood, "Enhanced myofibroblastic differentiation and survival in thy-1(-) lung fibroblasts," American Journal of Respiratory Cell and Molecular Biology, vol. 36, no. 2, pp. 226-235, 2007.

[17] G. Ramírez, J. S. Hagood, Y. Sanders et al., "Absence of Thy1 results in TGF- $\beta$ induced MMP-9 expression and confers a profibrotic phenotype to human lung fibroblasts," Laboratory Investigation, vol. 91, no. 8, pp. 1206-1218, 2011.

[18] J. S. Hagood, P. Prabhakaran, P. Kumbla et al., "Loss of fibroblast Thy-1 expression correlates with lung fibrogenesis," American Journal of Pathology, vol. 167, no. 2, pp. 365-379, 2005.

[19] J. Kitayama, S. Emoto, H. Yamaguchi, H. Ishigami, and T. Watanabe, "CD90(+) mesothelial-like cells in peritoneal fluid promote peritoneal metastasis by forming a tumor permissive microenvironment," PLoS ONE, vol. 9, no. 1, Article ID e86516, 2014.

[20] N. Jovanović, S. Zunić-Bozinovski, D. Trpinac et al., "Ultrastructural changes of the peritoneum in a rabbit model of peritoneal dialysis," Vojnosanitetski Pregled, vol. 70, no. 11, pp. 1023-1028, 2013.

[21] J. A. Jiménez-Heffernan, A. Aguilera, L. S. Aroeira et al., "Immunohistochemical characterization of fibroblast subpopulations in normal peritoneal tissue and in peritoneal dialysisinduced fibrosis," Virchows Archiv, vol. 444, no. 3, pp. 247-256, 2004.

[22] J. Loureiro, P. Sandoval, G. del Peso et al., “Tamoxifen ameliorates peritoneal membrane damage by blocking mesothelial to mesenchymal transition in peritoneal dialysis," PLOS ONE, vol. 8, no. 4, Article ID e61165, 2013.

[23] H. Seeger, N. Braun, J. Latus et al., "Platelet-derived growth factor receptor-beta expression in human peritoneum," Nephron Clinical Practice, vol. 128, no. 1-2, pp. 178-184, 2014.

[24] M. Mack and M. Yanagita, "Origin of myofibroblasts and cellular events triggering fibrosis," Kidney International, vol. 87, pp. 297-307, 2015.

[25] L. S. Aroeira, A. Aguilera, J. A. Sánchez-Tomero et al., "Epithelial to mesenchymal transition and peritoneal membrane failure in peritoneal dialysis patients: pathologic significance and potential therapeutic interventions," Journal of the American Society of Nephrology, vol. 18, no. 7, pp. 2004-2013, 2007.

[26] K. Honda, C. Hamada, M. Nakayama et al., "Impact of uremia, diabetes, and peritoneal dialysis itself on the pathogenesis of peritoneal sclerosis: a quantitative study of peritoneal membrane morphology," Clinical Journal of the American Society of Nephrology, vol. 3, no. 3, pp. 720-728, 2008.
[27] K. Shioshita, M. Miyazaki, Y. Ozono et al., "Expression of heat shock proteins 47 and 70 in the peritoneum of patients on continuous ambulatory peritoneal dialysis," Kidney International, vol. 57, no. 2, pp. 619-631, 2000.

[28] M. A. M. Mateijsen, A. C. van der Wal, P. M. E. M. Hendriks et al., "Vascular and interstitial changes in the peritoneum of CAPD patients with peritoneal sclerosis," Peritoneal Dialysis International, vol. 19, no. 6, pp. 517-525, 1999.

[29] L. Díaz-Flores, R. Gutiérrez, M. P. García et al., "CD34+ stromal cells/fibroblasts/fibrocytes/telocytes as a tissue reserve and a principal source of mesenchymal cells. Location, morphology, function and role in pathology," Histology and Histopathology, vol. 29, no. 7, pp. 831-870, 2014.

[30] M. Yáñez-Mó, E. Lara-Pezzi, R. Selgas et al., "Peritoneal dialysis and epithelial-to-mesenchymal transition of mesothelial cells," The New England Journal of Medicine, vol. 348, no. 5, pp. 403413, 2003.

[31] O. Devuyst, P. J. Margetts, and N. Topley, "The pathophysiology of the peritoneal membrane," Journal of the American Society of Nephrology, vol. 21, no. 7, pp. 1077-1085, 2010.

[32] Y. Liu, Z. Dong, H. Liu, J. Zhu, F. Liu, and G. Chen, “Transition of mesothelial cell to fibroblast in peritoneal dialysis: EMT, stem cell or bystander?" Peritoneal Dialysis International, vol. 35, no. 1, pp. 14-25, 2015.

[33] S. E. Mutsaers, K. Birnie, S. Lansley, S. E. Herrick, C. Lim, and C. M. Prele, "Mesothelial cells in tissue repair and fibrosis," Frontiers in Pharmacology, vol. 6, article 113, 2015.

[34] E. Sánchez-Tilló, Y. Liu, O. de Barrios et al., "EMT-activating transcription factors in cancer: beyond EMT and tumor invasiveness," Cellular and Molecular Life Sciences, vol. 69, no. 20, pp. 3429-3456, 2012.

[35] A. H. Yang, J. Y. Chen, and J. K. Lin, "Myofibroblastic conversion of mesothelial cells," Kidney International, vol. 63, no. 4, pp. 1530-1539, 2003.

[36] P. J. Margetts, P. Bonniaud, L. Liu et al., "Transient overexpression of TGF-betal induces epithelial mesenchymal transition in the rodent peritoneum," Journal of the American Society of Nephrology, vol. 16, no. 2, pp. 425-436, 2005.

[37] R. Strippoli, I. Benedicto, M. L. P. Lozano et al., "Inhibition of transforming growth factor-activated kinase 1 (TAK1) blocks and reverses epithelial to mesenchymal transition of mesothelial cells," PLoS ONE, vol. 7, no. 2, Article ID e31492, 2012.

[38] R. Strippoli, I. Benedicto, M. Foronda et al., "p38 maintains E-cadherin expression by modulating TAK1-NF- $\kappa$ B during epithelial-to-mesenchymal transition," Journal of Cell Science, vol. 123, no. 24, pp. 4321-4331, 2010.

[39] Q. Liu, Y. Zhang, H. Mao et al., "A crosstalk between the Smad and JNK signaling in the TGF- $\beta$-induced epithelialmesenchymal transition in rat peritoneal mesothelial cells," PLoS ONE, vol. 7, no. 2, Article ID e32009, 2012.

[40] Y.-H. Jang, H.-S. Shin, H. Sun Choi et al., "Effects of dexamethasone on the TGF-betal-induced epithelial-to-mesenchymal transition in human peritoneal mesothelial cells," Laboratory Investigation, vol. 93, no. 2, pp. 194-206, 2013.

[41] R. Strippoli, I. Benedicto, M. L. P. Lozano, A. Cerezo, M. LópezCabrera, and M. A. Del Pozo, "Epithelial-to-mesenchymal transition of peritoneal mesothelial cells is regulated by an ERK/NFkappaB/Snaill pathway," Disease Models and Mechanisms, vol. 1, no. 4-5, pp. 264-274, 2008.

[42] P. Patel, Y. Sekiguchi, K.-H. Oh, S. E. Patterson, M. R. J. Kolb, and P. J. Margetts, "Smad3-dependent and -independent 
pathways are involved in peritoneal membrane injury," Kidney International, vol. 77, no. 4, pp. 319-328, 2010.

[43] R. Vargha, M. Endemann, K. Kratochwill et al., "Ex vivo reversal of in vivo transdifferentiation in mesothelial cells grown from peritoneal dialysate effluents," Nephrology Dialysis Transplantation, vol. 21, no. 10, pp. 2943-2947, 2006.

[44] C. Li, Y. Ren, X. Jia et al., “Twist overexpression promoted epithelial-to-mesenchymal transition of human peritoneal mesothelial cells under high glucose," Nephrology Dialysis Transplantation, vol. 27, no. 11, pp. 4119-4124, 2012.

[45] M.-A. Yu, K.-S. Shin, J. H. Kim et al., "HGF and BMP7 ameliorate high glucose-induced epithelial-to-mesenchymal transition of peritoneal mesothelium," Journal of the American Society of Nephrology, vol. 20, no. 3, pp. 567-581, 2009.

[46] T. Y. H. Wong, A. O. Phillips, J. Witowski, and N. Topley, "Glucose-mediated induction of TGF-betal and MCP-1 in mesothelial cells in vitro is osmolality and polyol pathway dependent," Kidney International, vol. 63, no. 4, pp. 1404-1416, 2003.

[47] K. Bang, J. Jeong, J. H. Shin et al., "Heme oxygenase-1 attenuates epithelial-to-mesenchymal transition of human peritoneal mesothelial cells," Clinical and Experimental Nephrology, vol. 17, no. 2, pp. 284-293, 2013.

[48] D. J. Abraham, B. Eckes, V. Rajkumar, and T. Krieg, "New developments in fibroblast and myofibroblast biology: implications for fibrosis and scleroderma," Current Rheumatology Reports, vol. 9, no. 2, pp. 136-143, 2007.

[49] G. del Peso, J. A. Jimenez-Heffernan, M. A. Bajo et al., "Epithelial-to-mesenchymal transition of mesothelial cells is an early event during peritoneal dialysis and is associated with high peritoneal transport," Kidney International Supplements, vol. 73, no. 108, pp. S26-S33, 2008.

[50] P. J. Margetts, M. Kolb, L. Yu et al., "Inflammatory cytokines, angiogenesis, and fibrosis in the rat peritoneum," The American Journal of Pathology, vol. 160, no. 6, pp. 2285-2294, 2002.

[51] M. A. Bajo, M. L. Príez-Lozano, P. Albar-Vizcaino et al., "LowGDP peritoneal dialysis fluid ('balance') has less impact in vitro and ex vivo on epithelial-to-mesenchymal transition (EMT) of mesothelial cells than a standard fluid," Nephrology Dialysis Transplantation, vol. 26, no. 1, pp. 282-291, 2011.

[52] A. Fernández-Perpén, M. L. Pérez-Lozano, M.-A. Bajo et al., "Influence of bicarbonate/low-GDP peritoneal dialysis fluid (Bicavera) on in vitro and ex vivo epithelial-to-mesenchymal transition of mesothelial cells," Peritoneal Dialysis International, vol. 32, no. 3, pp. 292-304, 2012.

[53] I. Hirahara, Y. Ishibashi, S. Kaname, E. Kusano, and T. Fujita, "Methylglyoxal induces peritoneal thickening by mesenchymallike mesothelial cells in rats," Nephrology Dialysis Transplantation, vol. 24, no. 2, pp. 437-447, 2009.

[54] O. Busnadiego, J. Loureiro-Alvarez, P. Sandoval et al., "A pathogenetic role for endothelin-1 in peritoneal dialysis-associated fibrosis," Journal of the American Society of Nephrology, vol. 26, no. 1, pp. 173-182, 2015.

[55] R. Dixit, X. Ai, and A. Fine, "Derivation of lung mesenchymal lineages from the fetal mesothelium requires hedgehog signaling for mesothelial cell entry," Development, vol. 140, no. 21, pp. 4398-4406, 2013.

[56] Y. Li, J. Wang, and K. Asahina, "Mesothelial cells give rise to hepatic stellate cells and myofibroblasts via mesothelialmesenchymal transition in liver injury," Proceedings of the National Academy of Sciences of the United States of America, vol. 110, no. 6, pp. 2324-2329, 2013.
[57] S. Karki, R. Surolia, T. D. Hock et al., "Wilms' tumor 1 (Wt1) regulates pleural mesothelial cell plasticity and transition into myofibroblasts in idiopathic pulmonary fibrosis," The FASEB Journal, vol. 28, no. 3, pp. 1122-1131, 2014.

[58] Y.-T. Chen, Y.-T. Chang, S.-Y. Pan et al., "Lineage tracing reveals distinctive fates for mesothelial cells and submesothelial fibroblasts during peritoneal injury," Journal of the American Society of Nephrology, vol. 25, no. 12, pp. 2847-2858, 2014.

[59] N. Sakai, J. Chun, J. S. Duffield, T. Wada, A. D. Luster, and A. M. Tager, "LPA1-induced cytoskeleton reorganization drives fibrosis through CTGF-dependent fibroblast proliferation," The FASEB Journal, vol. 27, no. 5, pp. 1830-1846, 2013.

[60] T. A. Wynn and T. R. Ramalingam, "Mechanisms of fibrosis: therapeutic translation for fibrotic disease," Nature Medicine, vol. 18, no. 7, pp. 1028-1040, 2012.

[61] J. Witowski, A. Thiel, R. Dechend et al., "Synthesis of C-X-C and C-C chemokines by human peritoneal fibroblasts: induction by macrophage-derived cytokines," American Journal of Pathology, vol. 158, no. 4, pp. 1441-1450, 2001.

[62] J. Witowski, H. Tayama, K. Ksiek, M. Wanic-Kossowska, T. O. Bender, and A. Jörres, "Human peritoneal fibroblasts are a potent source of neutrophil-targeting cytokines: a key role of IL-1 $\beta$ stimulation," Laboratory Investigation, vol. 89, no. 4, pp. 414-424, 2009.

[63] E. Kawka, J. Witowski, N. Fouqet et al., "Regulation of chemokine CCL5 synthesis in human peritoneal fibroblasts: a key role of IFN- $\gamma$," Mediators of Inflammation, vol. 2014, Article ID 590654, 9 pages, 2014.

[64] C. Verger, A. Luger, H. L. Moore, and K. D. Nolph, "Acute changes in peritoneal morphology and transport properties with infectious peritonitis and mechanical injury," Kidney International, vol. 23, no. 6, pp. 823-831, 1983.

[65] B. Haslinger-Löffler, B. Wagner, M. Brück et al., "Staphylococcus aureus induces caspase-independent cell death in human peritoneal mesothelial cells," Kidney International, vol. 70, no. 6, pp. 1089-1098, 2006.

[66] D. Kitterer, J. Latus, C. Ulmer et al., "Activation of nuclear factor of activated T cells 5 in the peritoneal membrane of uremic patients," The American Journal of Physiology-Renal Physiology, vol. 308, no. 11, pp. F1247-F1258, 2015.

[67] R. S. Smith, T. J. Smith, T. M. Blieden, and R. P. Phipps, "Fibroblasts as sentinel cells. Synthesis of chemokines and regulation of inflammation," The American Journal of Pathology, vol. 151, no. 2, pp. 317-322, 1997. 


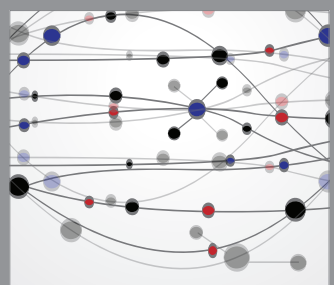

The Scientific World Journal
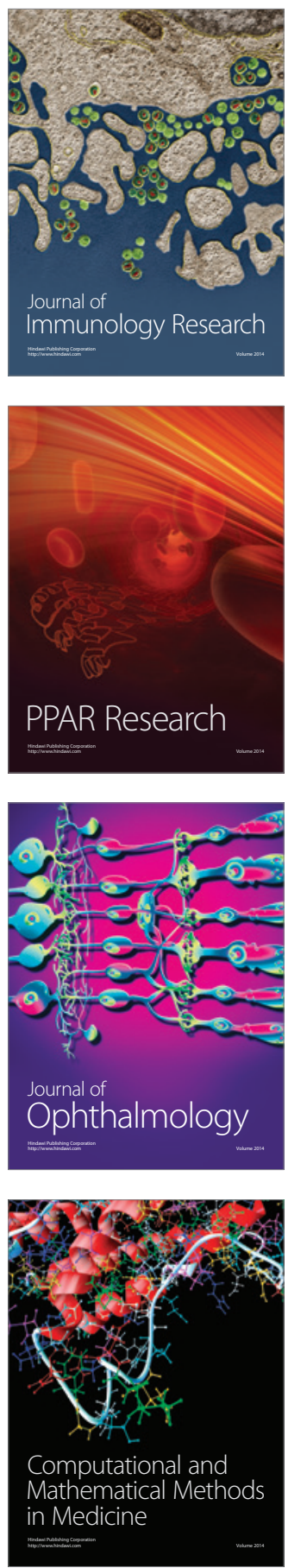

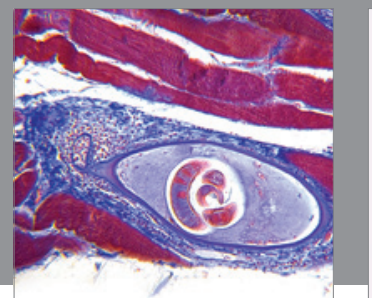

Gastroenterology

Research and Practice
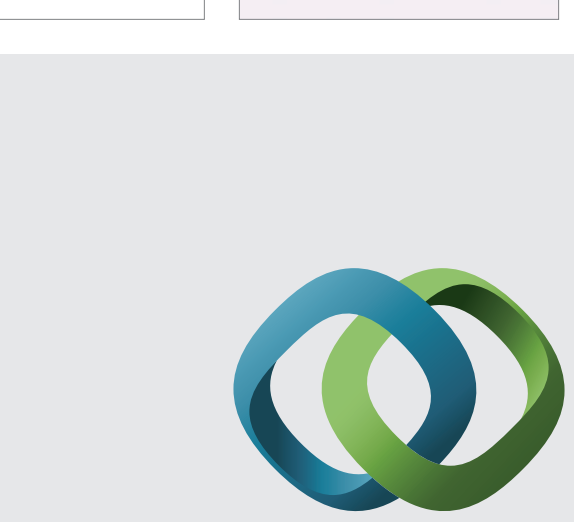

\section{Hindawi}

Submit your manuscripts at

http://www.hindawi.com
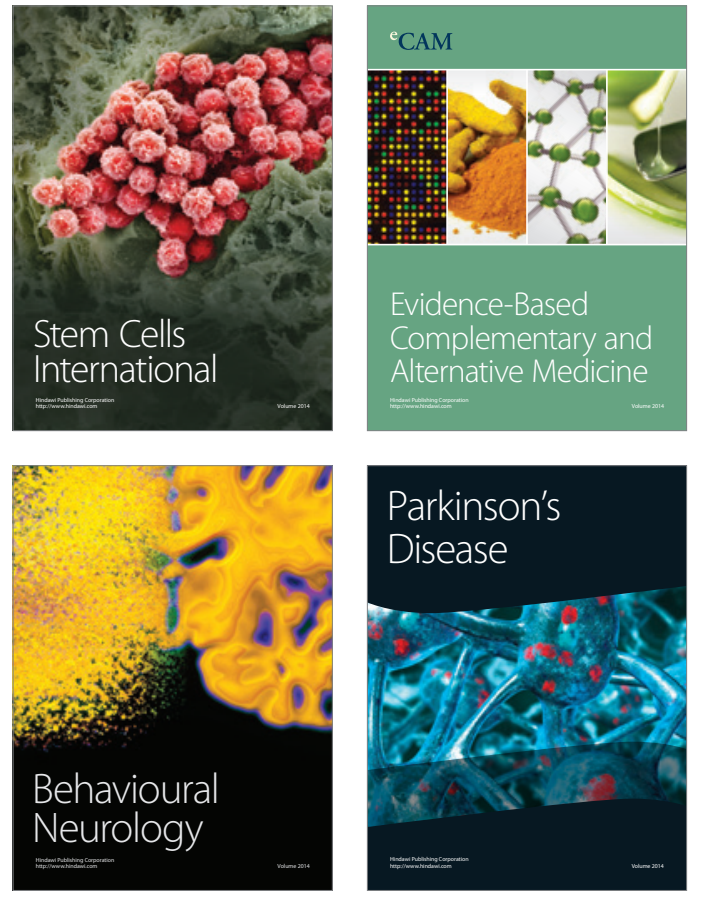
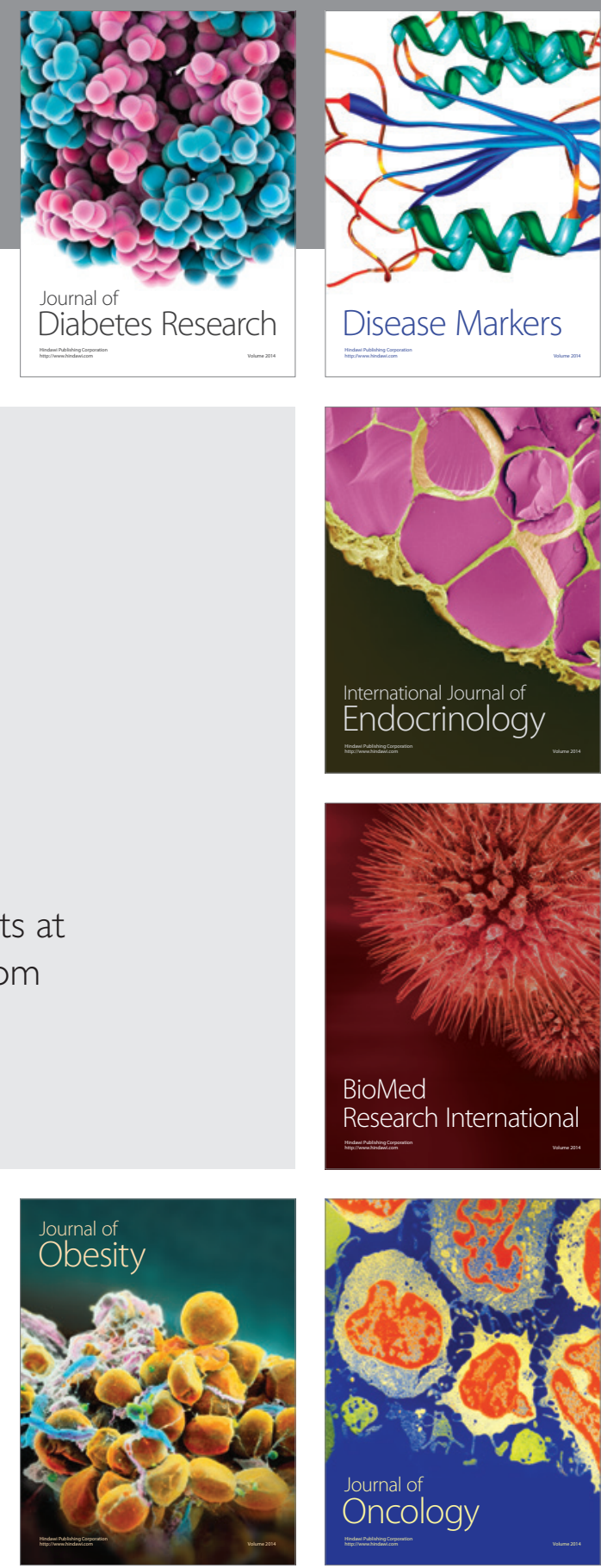

Disease Markers
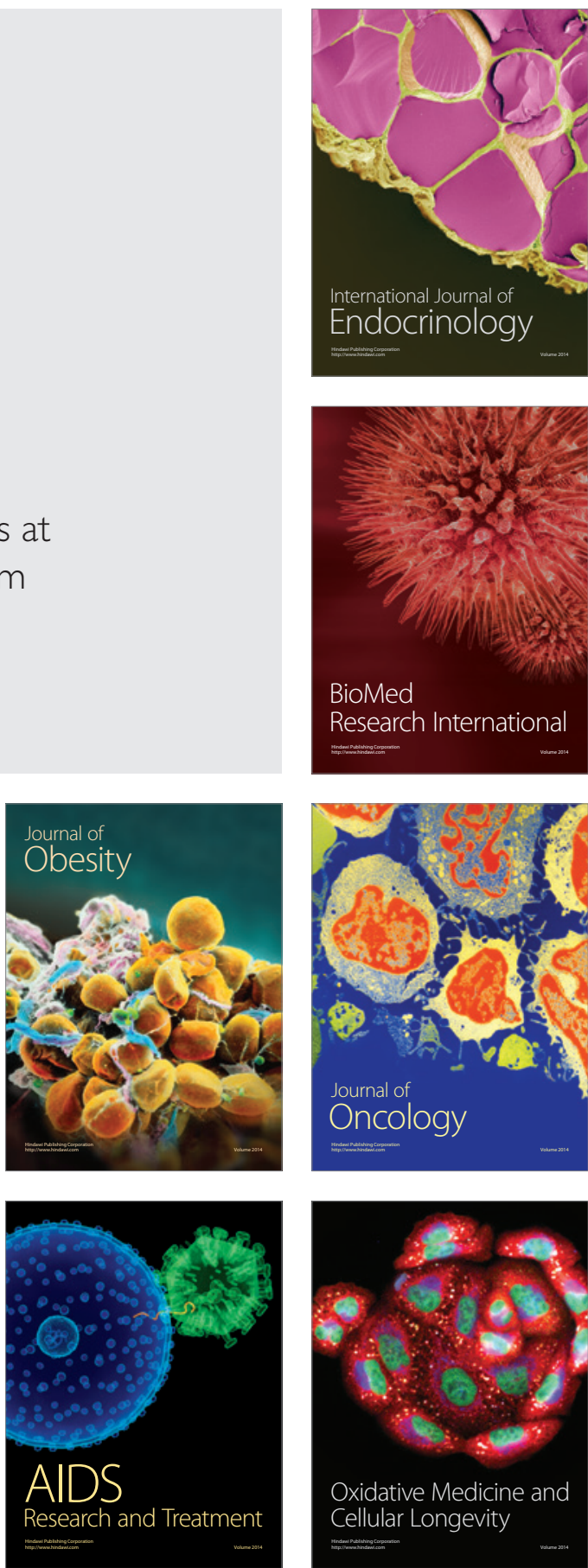